\title{
Impact of in situ physical and chemical cleaning on PVDF membrane properties and performances
}

\author{
M.F. Rabuni ${ }^{a}$, N.M. Nik Sulaiman ${ }^{a}$, M.K. Aroua ${ }^{a}$, Ching Yern Chee ${ }^{b}$, N. Awanis Hashim ${ }^{a, *}$ \\ a Department of Chemical Engineering, Faculty of Engineering, University of Malaya, 50603 Kuala Lumpur, Malaysia
}

b Department of Mechanical Engineering, Faculty of Engineering, University of Malaya, 50603 Kuala Lumpur, Malaysia

H I G H L I G H T S

- The effect of cleaning process at mild conditions towards PVDF membrane stability is investigated.

- Two commonly used cleaning agents ( $\mathrm{NaOH}$ and $\mathrm{NaOCl}$ ) were found to reduce PVDF membrane stability.

- The protein retention performance of membrane is reduced subsequent to membrane cleaning process.

- The stability of PVDF membrane was affected even at a chemical concentration of $0.01 \mathrm{M}$.

A R T I C L E IN F O

Article history:

Received 16 July 2014

Received in revised form

25 September 2014

Accepted 29 September 2014

Available online 14 October 2014

Keywords:

Poly(vinylidene fluoride)

Membrane cleaning

Chemical

$\mathrm{NaOH}$

$\mathrm{NaOCl}$

\begin{abstract}
A B S T R A C T
Appropriate selection of cleaning agent is an important factor to achieve a better cleaning efficiency and this topic has become an ongoing discussion. This work assesses the impacts of sodium hydroxide $(\mathrm{NaOH})$ and sodium hypochlorite $(\mathrm{NaOCl})$ aqueous solution towards polyvinylidene fluoride (PVDF) stability at the typical concentrations used in membrane cleaning. The cleaned membranes were characterised using field emission scanning electron microscopy (FESEM), Fourier transform infrared (FTIR), pure water flux measurement, contact angle, protein retention and tensile testing. Membrane cleaned at elevated temperature and higher concentration presented a higher water flux than the virgin membrane which can be a worrying sign of alteration in membrane properties. The FTIR spectra indicated that the alteration in chemical composition of the membrane causes a reduction in the degree of hydrophilicity. The mechanical properties of the membrane were compromised based on the declination of tensile strength. The findings from this work suggest that the usage of $\mathrm{NaOCl}$ as compared to $\mathrm{NaOH}$ causes a more detrimental effect towards the stability of the PVDF membrane.
\end{abstract}

c 2014 Elsevier Ltd. All rights reserved.

\section{Introduction}

At present, polyvinylidene fluoride (PVDF) has appeared as a favourable material for membrane fabrication due to its outstanding chemical, thermal and mechanical characteristics (Hashim et al., 2011; Liu et al., 2011; Zhang et al., 2009). However, pure PVDF exhibits high hydrophobicity which makes it easily fouled during the filtration process compared to hydrophilic material. For that reason, it is frequent to do modification on PVDF membrane to turn it into hydrophilic properties (Kim et al., 2009; Liu et al., 2011). One of the common methods to modify membrane is by the incorporation of hydrophilic additives during membrane preparation (Fontananova et al., 2006; Hajibabania et al., 2012). The additive is added to introduce hydrophilic functional group to

\footnotetext{
*Corresponding author. Tel.: +60 37967 5206; fax: +60 379675319
}

E-mail address: awanis@um.edu.my (N. Awanis Hashim).

http://dx.doi,org/10.1016/j.ces.2014.09.053

0009-2509/o 2014 Elsevier Ltd. All rights reserved. the structure that will aid water permeation. Despite its enhanced property, fouling is still unavoidable. Fouling could be described into two types, external and internal fouling. External fouling occurs when certain impurities or constituents known as foulants are deposited on the membrane surface. The internal fouling takes place by the adsorption and deposition of solutes and small particles within the internal structure of membranes, e.g., adsorption of foulants to pore-walls and pore narrowing or blocking (Wang et al., 2014). This phenomenon contribute to a decrement in membrane flux and permeability, thus lead to a poor separation performance (Puspitasari et al., 2010).

Since fouling remains an inevitable event, consequently, membrane cleaning is accepted as a compulsory procedure during the filtration operation in order to restore membrane flux and performance. There are several ways to clean membrane with each depending on the types of foulant (Hilal et al, 2005; Porcelli and Judd, 2010). The fouled membrane could be physically cleaned via backwashing or flushing. This method can potentially eliminate 
the cake layer that has been deposited on the surface layer. However, physical cleaning is ineffective in the case where foulants were adsorbed into the membrane layer or pore plugging by means of chemical reaction that occurred between the foulants and membrane material. Accordingly, chemical cleaning is the proper approach to remove irreversible foulant for optimum restoration of the flux.

There have been many studies conducted with regards of fouling, yet, only a small number of research were committed to study PVDF membrane ageing that are caused by chemical cleaning (Hajibabania et al., 2012; Levitsky et al., 2011; Puspitasari et al., 2010). Sodium hydroxide $(\mathrm{NaOH})$ and sodium hypochlorite $(\mathrm{NaOCl})$ are both commonly used in the membrane cleaning study (Kimura et al., 2004; Lin et al, 2010; Regula et al., 2013: Suárez et al., 2012; Wang et al., 2010). For instance, caustic solution was found to be efficient in restoring flux of membrane that had been fouled with humic acid (HA) (Srisurichan et al., 2005). Cleaning was also performed with aqueous $\mathrm{NaOH}(1 \mathrm{wt} \%)$ on a submerged rotating membrane bioreactor system that was severely fouled (Zuo et al., 2010). Subsequent to cleaning, distinct membrane pores can be observed given that the alkaline solution successfully removes the organic contaminants of protein, together with the microorganisms that lie inside and on the surface of the membrane. Levitsky et al. evaluated the effects of cleaning a bovine serum albumin (BSA) fouled PVDF membrane using $\mathrm{NaOCl}$ (Levitsky et al., 2011). In general, all cleaned membranes presented a higher permeability than the virgin membranes which implies an enhanced hydrophilicity of the membrane. In another study, hydrochloric acid $(\mathrm{HCl}), \mathrm{NaOH}$ and $\mathrm{NaOCl}$ were used for cleaning process of membrane fouled with coke particles (Madaeni et al., 2011). The result shows both $\mathrm{NaOH}$ and $\mathrm{NaOCl}$, performed a flux recovery of more than $75 \%$ while $\mathrm{HCl}$ produced a lesser result. Other than analysing the efficacy of chemical cleaning for membrane water flux recovery, the changes in polymeric membrane stability following the cleaning process was also studied (Arkhangelsky et al., 2007). It was found that the membrane exposed to hypochlorite experienced a decline in its elasticity, tensile strength, and elongation at break. An intriguing remark was made by the author whereby a reduction in mechanical strength was detected even at hypochlorite concentration that is considered as "safe-cleaning" conditions. In our recent study, we too, showed the exposure to strong alkaline solution even at mild condition could be detrimental to PVDF membrane (Rabuni et al., 2013).

All of the previous works mentioned above have demonstrated that the chemical cleaning to be relatively satisfactory in recovering membrane flux. The efficiency of cleaning has been mostly discussed with regards to its flux recovery and rejection performance rather than to scrutinise the effects of the chemical used towards the stability of the membrane material itself. Thus, it has not been realised that the membrane deteriorates throughout the cleaning process. There have long been suspicions that $\mathrm{NaOH}$ and $\mathrm{NaOCl}$ could be detrimental to PVDF. However, not many works had been done to investigate the effect of these cleaning agents on PVDF membrane. To our knowledge, discussions on the effects of chemical exposure on the PVDF membrane intrinsic properties primarily in repetitive fouling and cleaning cycles are only available in limited references (Hajibabania et al., 2012; Puspitasari et al., 2010; Wang et al., 2010). It was observed that cyclical filtration and cleaning has changed the PVDF membrane stability. Previous research usually involved a longer cleaning time and relatively high dose of chemical exposed to the fouled membrane. Furthermore, there is a lack of detailed study on the comparison of $\mathrm{NaOH}$ and $\mathrm{NaOCl}$ used to clean fouled PVDF membrane. The compatibility of cleaning agents and the membrane material is vital to ensure a longer and improved membrane lifespan. Therefore, in this work, we aim to investigate the effect of repeated chemical cleaning of fouled PVDF membranes analogous to cyclical chemical cleaning processes for membrane system in real industry-related application. It is also worth to state that the main difference between this and earlier studies is the application of the comparatively mild-cleaning conditions i.e. shorter cleaning time and relatively lower chemical concentration.

\section{Experimental}

\subsection{Materials}

Commercial flat sheet ultrafiltation (UF) PVDF membrane with $100 \mathrm{~K}$ molecular weight cut-off (MWCO) was purchased from Sterlitech Corporation (USA). Due to the proprietary nature of the commercial membrane formulations, information on the type of hydrophilic additive used for PVDF modification cannot be disclosed. Throughout this work, ultrapure water (Milli-Q) was used for rinsing, preparation of sample solution and membrane water flux measurement $\mathrm{NaOCl}$ with $4.99 \%$ available chlorine was obtained from Fisher Scientific (Malaysia) and $\mathrm{NaOH}$ was purchased from R\&M Chemicals (Malaysia). Bovine serum albumin (BSA) in a form of lyophilised powder with a molecular weight of approximately $66 \mathrm{kDa}$ was purchased from Sigma Aldrich (Malaysia). The protein solution was prepared by mixing the BSA solid powder in Milli-Q water.

\subsection{Methodology}

The filtration experiments were conducted with the cross-flow filtration module (Solteq membrane bench filtration (Model TR 32), Malaysia). Prior to filtration experiment, the membrane was rinsed to remove any preservative on its surface and soaked in Milli-Q water for approximately one day. A sequence of experiments was carried out as depicted in Fig. 1.

Throughout this study, the cleaning process was performed insitu (without removing the membrane from the module). Physical cleaning of the fouled membrane was conducted by means of hydraulic via forward flush. The ultrapure water was allowed to flow along the membrane for 20 min with a velocity of approximately $5 \mathrm{~m} / \mathrm{min}$. In principle, this process could remove a constructed layer of contaminants on the membrane surface through the creation of turbulence. Afterwards, cleaning with chemical was conducted in similar approach, by allowing the cleaning solution to flow across the fouled membrane surface at different cleaning conditions. The parameters in chemical cleaning procedure were selected in accordance with the commonly employed membrane cleaning conditions (i.e. chemical concentration, cleaning period and temperature) documented in earlier works (Chen et al., 2006; Regula et al., 2014) and also based on the recommended cleaning protocol/guidelines by several membrane manufacturers. In this work, three different concentrations were tested $(0.01,0.05$ and $0.1 \mathrm{M}$ ) and the cleaning period were $10,20,40$ and $60 \mathrm{~min}$. The effect of different temperature on cleaning performance was investigated by cleaning the membrane at room condition $\left(25 \pm 2{ }^{\circ} \mathrm{C}\right)$ and $50 \pm 2{ }^{\circ} \mathrm{C}$.

For comparison purposes, membrane cleaning was conducted with both $\mathrm{NaOH}$ and $\mathrm{NaOCl}$. After the chemical cleaning process, the filtration system was rinsed for $15 \mathrm{~min}$ with pure water. During oxidative $(\mathrm{NaOCl})$ cleaning experiments, a little amount of sodium bisulphite was added to remove traces of $\mathrm{NaOCl}$ prior to another cycle of BSA filtration. To evaluate the flux recovery, the ratio of the specific flux $\left(\mathrm{L} \mathrm{m}^{-2} \mathrm{~h}^{-1} \mathrm{bar}^{-1}\right)$ of permeate flux for cleaned membrane, $J_{c}$, to the initial flux of virgin membrane, $J_{v}$, was calculated and described as cleaning efficiency $\left(C_{e}\right)$ and is 


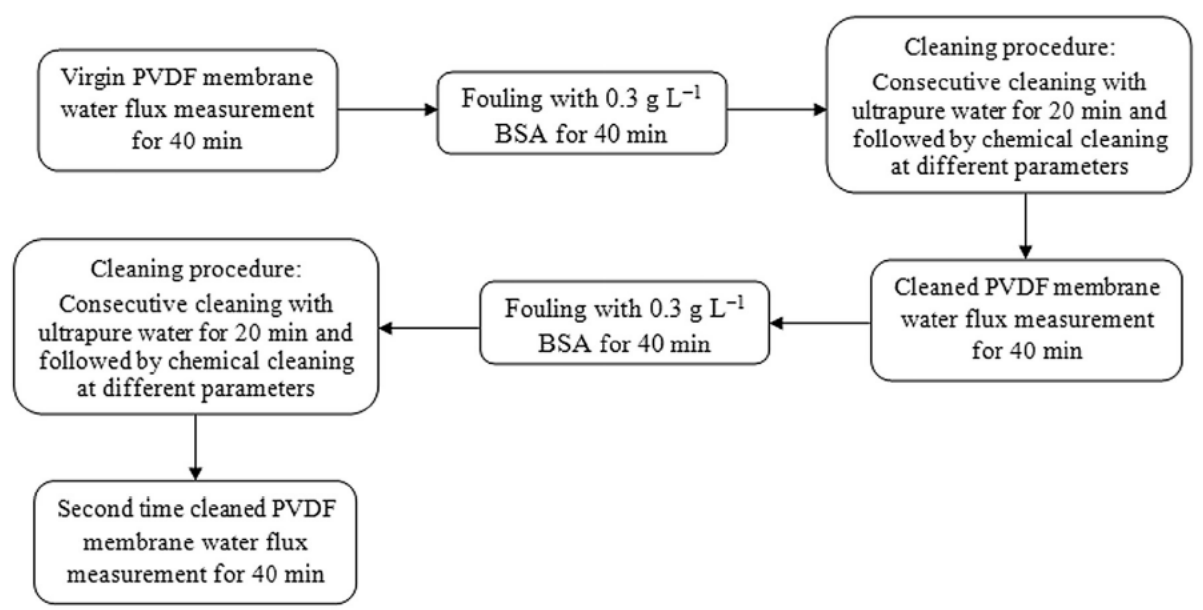

Fig. 1. Experimental works for repetitive fouling and cleaning.

represented using the following equation;

Cleaning efficiency $\left(C_{e}\right)=\frac{J_{c}}{J_{v}}$

\subsection{Membrane characterisation technique}

The membrane permeability was assessed by water flux measurement. A series of membrane water flux measurement and protein filtration were done using the cross-flow filtration module with effective membrane working area of $41.0 \mathrm{~cm}^{2}$. The flux of Milli- $Q$ water across the membrane was measured at a transmembrane pressure (TMP) of one bar. The changes in PVDF membrane properties were analysed through Field emission scanning electron microscope (FESEM), Fourier transform infrared (FTIR), contact angle measurement, protein retention and tensile test. The morphology of the PVDF membrane surface was inspected using FESEM (Carl Zeiss Microscopy, North America) The dried samples were placed on a pin stub mount and are then coated with gold for $30 \mathrm{~s}$. The images of the surface of the virgin and treated membranes were taken at a range of various magnifications. The chemical composition of PVDF membranes was analysed by attenuated total reflectance Fourier transform infrared (ATR-FTIR) (Nicolet iS10 Thermo Scientific, USA). The spectra were measured at wavenumbers in the range of $650-4000 \mathrm{~cm}^{-1}$. Dataphysics OCA-15 plus (DataPhysics, USA) was used to measure membrane contact angle using the sessile drop method. In this test, a $20 \mu \mathrm{l}$ drop of distilled water was positioned onto the surface of the dried membrane using a micro syringe, and the measurement of air-water-surface contact angle was done within $10 \mathrm{~s}$. At least five measurements were done using separate pieces of membrane. BSA protein retention was measured in accordance with the Bradford assay method (Bradford, 1976). The graph of absorbance at $595 \mathrm{~nm}$ against BSA protein concentration was plotted and a linear line was constructed. The measurement of BSA available in the filtration permeate was made by referring to the corresponding value of $x$-axis (concentration of BSA) of the standard curve to the absorbance at $595 \mathrm{~nm}$ ( $y$-axis). All measurements were done by at least four replicates. The monitoring of adsorption peak at $595 \mathrm{~nm}$ was done with a UV-vis spectrometer. The BSA retention percentage was calculated using the following relation:

1) $\quad$ BSA retention $=\left(1-\frac{C_{p}}{C_{f}}\right) \times 100 \%$

where $C_{p}$ and $C_{f}$ are the BSA concentration in the permeate and in the feed, respectively. Instron 5569 tensile testing machine (Instron, USA) was used to measure the membrane tensile strength. Tensile testing of the sample was done in accordance with ASTM D882. Each sample was cut into similar shape prior to the testing. The sample was secured at both ends and pulled at a constant elongation rate of $50 \mathrm{~mm} \mathrm{~min}{ }^{-1}$ with an initial gauge length of $8 \mathrm{~cm}$. To minimise the experimental error, the tensile test was performed five times for each sample with a standard deviation of less than $5 \%$.

\section{Results and discussion}

\subsection{Membrane water flux measurement}

As a preliminary study, the cleaning process was done by means of physical cleaning since some foulants might probably be detached by hydraulic means. Physical cleaning was found to be ineffective to recover water flux since the result was only approximately 33\% of the cleaning efficiency as shown in Fig. 2. This low efficiency is believed to be caused by a strong adsorption of BSA particle to the membrane surface, wherein the strong bond could only be broken by means of chemical attack or reaction. Hence, the cleaning with chemical agent is an ideal option for protein removal. Despite the low efficiency of physical cleaning, it was performed with the intention to loosen the interactions of BSA particle deposited on the membrane. The target for cleaning efficiency was set to 1 which means that the flux for cleaned membrane should be comparable to the flux of the virgin membrane. It has been generally accepted that higher concentration of chemical agent will contribute to a better cleaning on the fouled membrane.

Fig. 3 depicts the effect of various chemical concentrations towards cleaning efficiency. As found in this study, the cleaning effect of either $\mathrm{NaOH}$ or $\mathrm{NaOCl}$ increases when their concentration is increased. This trend is similar to the membrane cleaning performance recorded in previous works (Puspitasari et al., 2010; 


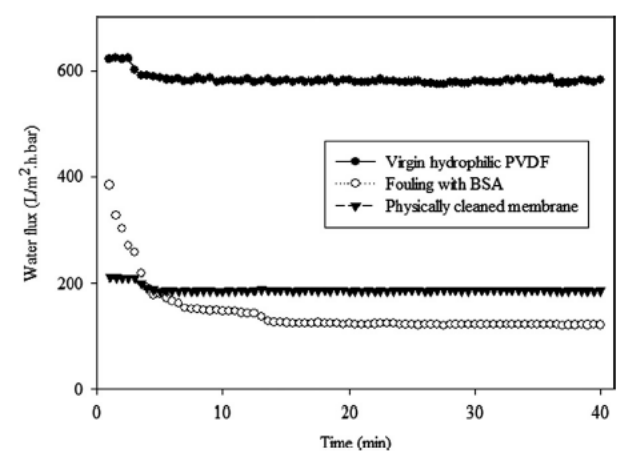

Fig. 2. Physically cleaned membrane water flux.

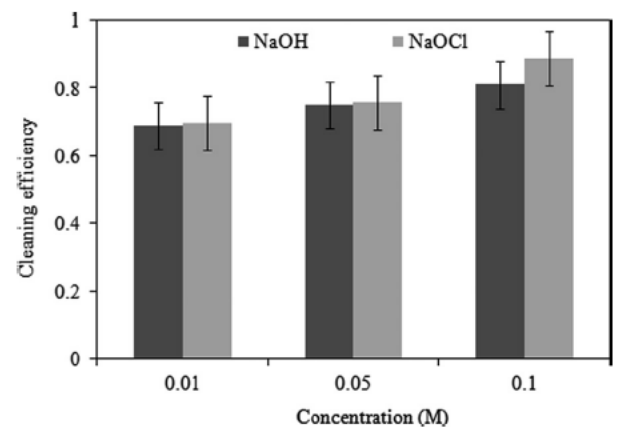

Fig. 3. Cleaning efficiency of membranes cleaned at different chemical concentration for $60 \mathrm{~min}$ at room temperature.

Wang et al., 2013). The lower concentration resulted in a relatively lower cleaning efficiency in comparison to cleaning with $0.1 \mathrm{M}$ of the solutions which contributed to a flux restoration of approximately $81 \%$ and $88 \%$ from the initial flux for $\mathrm{NaOH}$ and $\mathrm{NaOCl}$ cleaned membranes, respectively.

As suggested in the technical manual provided by one of the membrane manufacturers (Dow, USA), the temperature of the cleaning solutions must be more than $25^{\circ} \mathrm{C}$ for maximum effectiveness. Elevating the temperature of the cleaning solution will assist in organic removal from the membrane surface. The effect of temperature on cleaning efficiency is depicted in Fig. 4 which shows that higher temperature improves the merit of cleaning performance.

At room temperature, the maximum cleaning efficiency was recorded to be approximately 0.81 and 0.88 using $\mathrm{NaOH}$ and $\mathrm{NaOCl}$, respectively whilst a significantly higher flux recovery was observed for a similar cleaning time at $50{ }^{\circ} \mathrm{C}$. At a higher cleaning temperature, during 20-40 min of the cleaning process, a comparable cleaning efficiency was recorded to be in the range of 0.890.94 . The cleaning efficiency was further improved with a cleaning process of $60 \mathrm{~min}$ at which the measured water flux was recorded to be higher than the virgin membrane. The flux recovery of more than $100 \%$ could be a potential remark of the alteration in membrane characteristic and probable membrane degradation (Hajibabania et al., 2012).

Notwithstanding an increase in cleaning effectiveness, a higher chemical concentration employed was liable for a more severe future fouling. The fouling tendency of the membranes was observed from the decline in water flux permeability during

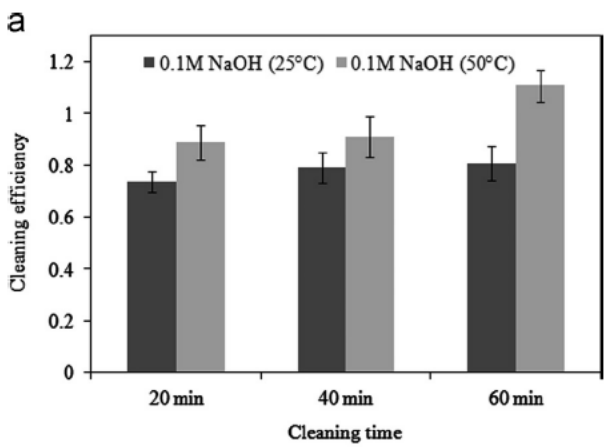

b

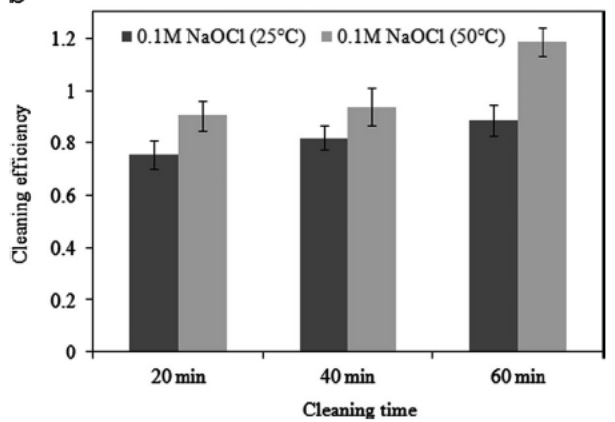

Fig. 4. Cleaning efficiency for membrane cleaned with (a) $0.1 \mathrm{M} \mathrm{NaOH}$ and (b) $0.1 \mathrm{M} \mathrm{NaOCl}$ at different temperatures.
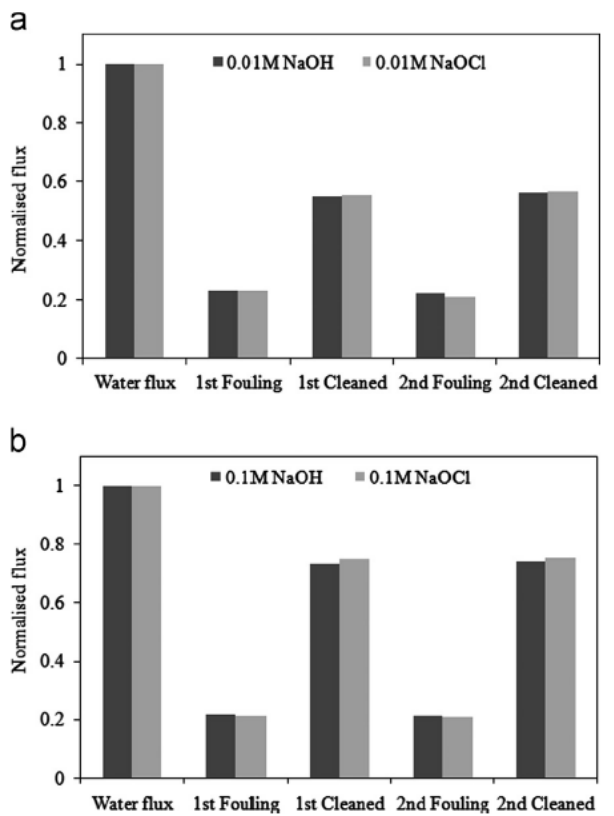

Fig. 5. Effect of repetitive cleaning on membrane water flux for $20 \mathrm{~min}$ cleaning time using (a) $0.01 \mathrm{M}$ and (b) $0.1 \mathrm{M}$ of chemical concentration. 
ultrafiltration of BSA solution. As presented in Fig. 5, it was shown that the cleaning agent's concentration is the major contributor in the upcoming fouling. During the second cycle of BSA filtration, the cleaned membrane was observed to experience a further decline in flux as represented by the second fouling. The degree of fouling becomes greater once a higher concentrated solution of cleaning agent is being used. Generally, a $\mathrm{NaOCl}$ cleaned membrane displays a better flux recovery than a $\mathrm{NaOH}$ cleaned membrane. However, when the cleaned membrane was subjected to another BSA filtration, the membrane which was cleaned by the former experienced a more severe fouling. For instance, during the cleaning of the membrane with $0.01 \mathrm{M} \mathrm{NaOCl}$, the normalise flux for first fouling was recorded to be at 0.23 and reduced to 0.21 during the second fouling cycle, whereas when $0.01 \mathrm{M} \mathrm{NaOH}$ was used, it showed only a slight reduction from 0.23 to 0.22 .

It was previously alleged that chemical cleaning improves the degree of PVDF membrane hydrophilicity (Hajibabania et al., 2012 Levitsky et al., 2011), thus, giving higher water flux. Conversely, the contrary outcome was observed in our work as membranes became less hydrophilic (based on contact angle values) afte cleaning. Further discussion on contact angle measurement is explained in Section 3.4. Subsequent to the first cleaning process,
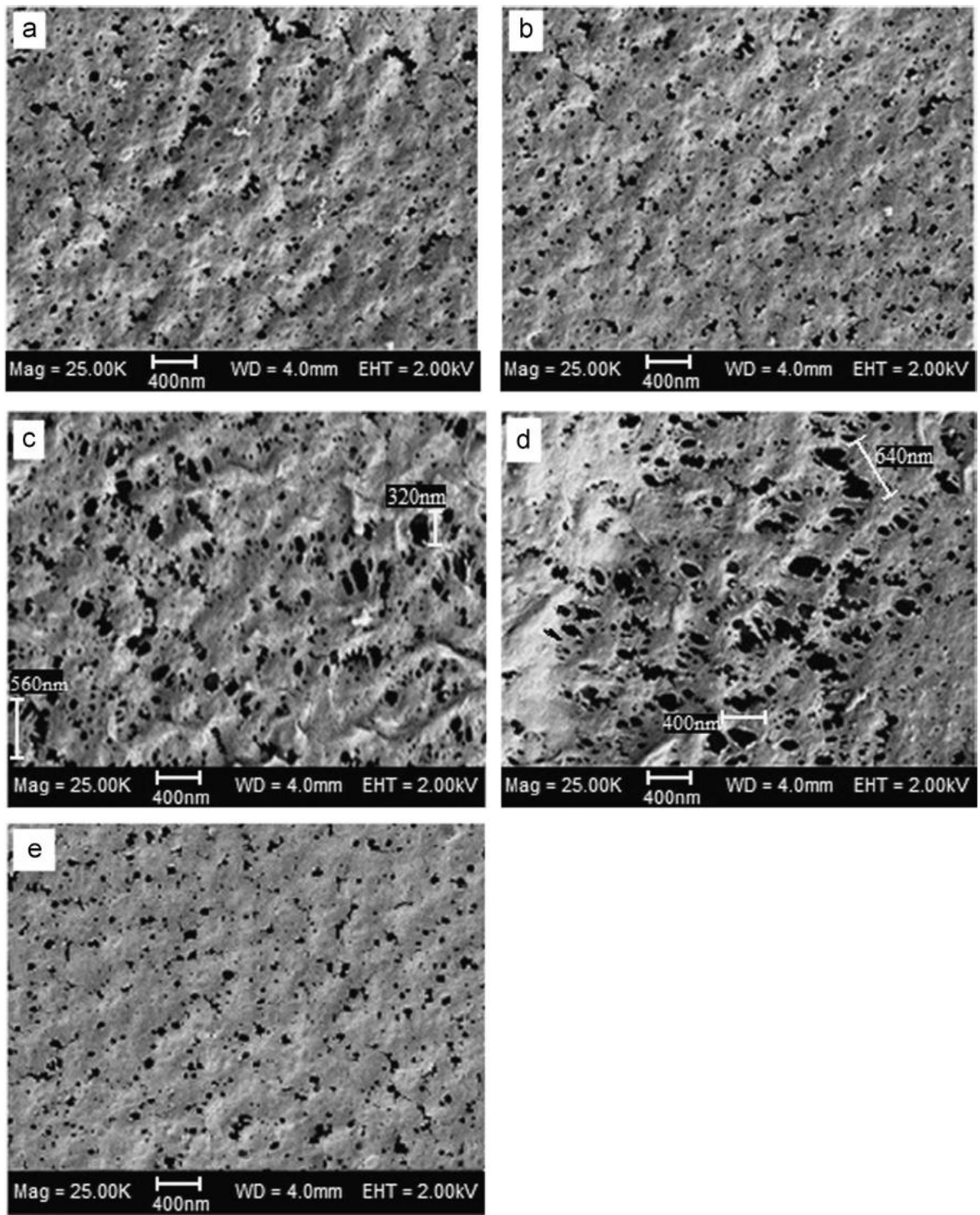

Fig. 6. FESEM images with 25,000 magnification of membrane surface area cleaned at room temperature by: (a) $0.01 \mathrm{M} \mathrm{NaOH}$, (b) $0.01 \mathrm{M} \mathrm{NaOCl}$, (c) $0.1 \mathrm{M} \mathrm{NaOH}$, (d) $0.1 \mathrm{M}$ $\mathrm{NaOCl}$ and (e) virgin membrane. 
the membrane was determined to exhibit higher contact angle values. This result could be explained by the instability of the PVDF modified membrane to retain its hydrophilicity.

Pure PVDF is indeed hydrophobic by nature. Through surface modification, PVDF could be turned into a hydrophilic membrane. Commercial membrane is usually made with the addition of hydrophilic additive to its casting solution purposely for pore forming and surface modification (Liu et al., 2011). The reduced hydrophilicity contributes to the inclination of BSA to foul on the membrane surface as a more severe fouling can be observed during the second cycle of protein filtration. The reason of this event is supported by the fact that hydrophobic membranes (in this particular work, cleaned membrane became less hydrophilic than the virgin membrane) have been recognised to be more susceptible to fouling by organic material. These findings signify that during cleaning, the cleaning agent attacks the structure of membrane which causes the alteration in hydrophilicity of the membrane.

In general, $\mathrm{NaOCl}$ offers a better cleaning performance to restore the membrane flux and permeability than that of $\mathrm{NaOH}$. The higher cleaning efficiency by the oxidant is contributed by the effective oxidation in the layer of adsorbed organic macromolecules which is close to the membrane surface (Kuzmenko et al., 2005). Taken as a whole, chemical cleaning has demonstrated its capability to eliminate BSA from the membrane surface. However, the exposure of chemicals to PVDF membrane during cleaning affects the membrane hydrophilicity which leads to a more severe fouling.

\subsection{Morphology of PVDF flat membranes}

The morphology and structure of the virgin and cleaned membranes were inspected using FESEM analysis and the images were illustrated in Fig. 6. From Fig. 6, the membrane which was cleaned with either $\mathrm{NaOCl}$ or $\mathrm{NaOH}$ showed almost no trace of BSA deposition on the membrane surface. This may generally suggest that cleaning using chemical is capable of removing foulant from the membrane surface. When $0.01 \mathrm{M}$ of these cleaning agents was used, the cleaned membrane displayed no significant changes in pore sizes as depicted in Fig. 6(a) and (b). Noticeable changes in membrane pore structure could be observed after higher chemical concentration was used during the cleaning procedure. As shown in Fig. 6(c) and (d), membranes which were cleaned with $0.1 \mathrm{M}$ chemical solution experienced more destruction as indicated by the apparent pore enlargement. It could be estimated from the scale of the FESEM image that the pore sizes of $\mathrm{NaOCl}$ cleaned membrane to be relatively larger than the $\mathrm{NaOH}$ cleaned one. The enlargement of membrane pore can be linked to the polymer chain breakage (Rabuni et al., 2013). In the case which involves PVDF material, it undergoes a dehydrofluorination reaction once it is exposed to the alkaline environment (Hashim et al., 2011).

\subsection{FTIR analysis}

The typical wavenumber for PVDF membrane shows the appearance of the characteristic bands at 762, 796, 875, 974, $1070,1181 \mathrm{~cm}^{-1}$ which is similar to PVDF film containing $\alpha$-phase, whilst several other peaks detected at 841,1274 and $1403 \mathrm{~cm}^{-1}$ are believed to be attributed by the $\beta$-phase (Gregorio, 2006; Hashim et al., 2011). From previous works, the occurrence of dehydrofluorination process largely indicated by the presence of the peaks at a range of $1590-1650 \mathrm{~cm}^{-1}$ assigned for carboncarbon double bond, $1700-1800 \mathrm{~cm}^{-1}$ assigned for carbonyl bond and $2100 \mathrm{~cm}^{-1}$ represents the carbon-carbon triple bond (Hashim et al., 2011; Kise and Ogata, 1983; Zhang et al., 2006). Any changes occurred in PVDF chemical composition could be largely detected

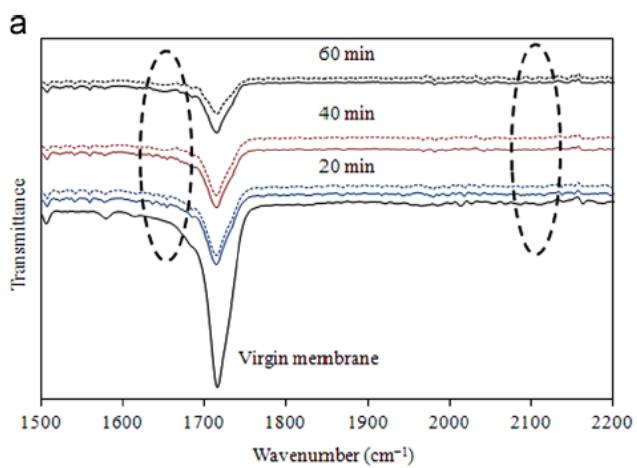

b

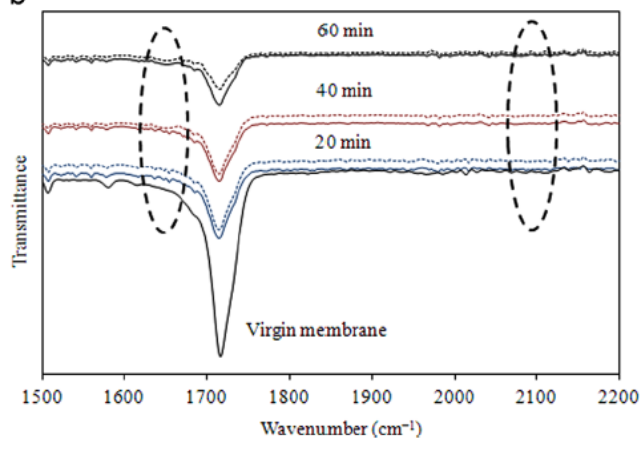

Fig. 7. Spectra of membrane cleaned at different time with (a) $\mathrm{NaOH}$ and (b) NaOCl; solid line represents $0.01 \mathrm{M}$ and dashed line represents $0.1 \mathrm{M}$ chemical solutions.

by the appearance of carbon-carbon double and triple bond (Ross et al., 2000). The spectra of membranes cleaned at various times are presented in Fig. 7. For a period of 20 min cleaning time, the spectra of all cleaned membranes showed a similar pattern regardless of the chemical concentration used. At a longer cleaning time, the FTIR analysis showed changes which occurred in chemical groups on the membrane surface.

Particularly for PVDF membranes, the alteration in chemical composition can be associated with the dehydrofluorination reaction. The dehydrofluorination process was mainly indicated by the occurrence of the peaks at a range of $1590-1650 \mathrm{~cm}^{-1}$ assigned for carbon - carbon double bond and $2100 \mathrm{~cm}^{-1}$ for the carbon-carbon triple bond (Hashim et al., 2011; Kise and Ogata, 1983). It was observed that a small peak at $1650 \mathrm{~cm}^{-1}$ (assigned for carbon-carbon double bond) appeared for membranes that were cleaned using $0.1 \mathrm{M}$ of either $\mathrm{NaOH}$ or $\mathrm{NaOCl}$ for a lapse of 40 and $60 \mathrm{~min}$, and membranes that were cleaned with $0.01 \mathrm{M}$ for $60 \mathrm{~min}$. The appearance of carbon-carbon double bond denoted the occurrence of dehydrofluorination reaction on the cleaned membrane surface (Rabuni et al,, 2013; Zhao et al, 2011). Although the peak signal was relatively low, its appearance signifies that there was an alteration in chemical composition when membranes were cleaned using the chemicals. In all cases, no peak indicating carbon-carbon triple bond was observed.

In addition, an apparent change in the relative intensity was observed for a peak at approximately $1710 \mathrm{~cm}^{-1}$ (assigned for $\mathrm{C}=\mathrm{O}$ ). The relative intensity for cleaned membrane was reduced significantly as compared to the virgin membrane. We believe that the peak representing carbonyl bond $(\mathrm{C}=0)$ is attributed by the

\section{Link to Full-Text Articles :}

\section{http://www.sciencedirect.com/science/article/pii/S0009250914005661}

\section{http://repository.um.edu.my/95281/1/Fairus\%20ChemEngSci\%202014.pdf}

